\title{
Artefact
}

Techniques, histoire et sciences humaines

Musées éphémères, musées imaginaires, musées perdus

\section{Mathieu Flonneau, Léonard Laborie et Arnaud Passalacqua (dir.), Les transports de la démocratie. Approche historique des enjeux politiques de la mobilité Rennes, Presses universitaires de Rennes, 2014}

\section{Konstantinos Chatzis}

\section{OpenEdition}

\section{Journals}

\section{Édition électronique}

URL : http://journals.openedition.org/artefact/732

DOI : $10.4000 /$ artefact.732

ISSN : 2606-9245

Éditeur :

Association Artefact. Techniques histoire et sciences humaines, Presses universitaires du Midi

\section{Édition imprimée}

Date de publication : 15 juin 2017

Pagination : 221-224

ISBN : 978-2-7535-6525-8

ISSN : 2273-0753

\section{Référence électronique}

Konstantinos Chatzis, « Mathieu Flonneau, Léonard Laborie et Arnaud Passalacqua (dir.), Les transports de la démocratie. Approche historique des enjeux politiques de la mobilité ", Artefact [En ligne], 5 | 2016, mis en ligne le 15 novembre 2017, consulté le 24 septembre 2020. URL : http:// journals.openedition.org/artefact/732 ; DOI : https://doi.org/10.4000/artefact.732

\section{Ce document a été généré automatiquement le 24 septembre 2020}

Artefact, Techniques, histoire et sciences humaines est mise à disposition selon les termes de la Licence Creative Commons Attribution - Pas d'Utilisation Commerciale - Pas de Modification 4.0 International. 


\section{Mathieu Flonneau, Léonard Laborie et Arnaud Passalacqua (dir.), Les transports de la démocratie. Approche historique des enjeux politiques de la mobilité}

Rennes, Presses universitaires de Rennes, 2014

Konstantinos Chatzis

\section{RÉFÉRENCE}

Mathieu Flonneau, Léonard Laborie et Arnaud Passalacqua (dir.), Les transports de la démocratie. Approche historique des enjeux politiques de la mobilité, Rennes, Presses universitaires de Rennes, 2014, $222 \mathrm{p}$.

1 Dirigé par trois historiens, Les transports de la démocratie se démarque d'un livre d'histoire pour plusieurs raisons. Si la discipline est bien représentée par plusieurs interventions, on ne manque pas de trouver parmi les contributeurs des politistes, des géographes-aménageurs et des économistes. Et, s'il est vrai que le présent s'immisce dans tout questionnement historique, force est également de constater que le dialogue entre la situation actuelle et les époques passées est plus intense et plus explicitement articulé dans cet ouvrage que dans la plupart des livres d'histoire: comme les coordinateurs l'annoncent dans l'introduction générale, le livre qu'ils ont dirigé a certes une "destination plutôt historique», mais celle-ci «ne doit pas masquer la vocation d'être utile aux citoyens de nos sociétés troublées » (p. 14).

2 Troublées par quoi? Par un «true and deep paradigm shift in assessement and connotation of mobility and democracy» (p. 10), à en croire l'historien Hans-Liudger Dienel qui préface le volume. Ce changement de paradigme ne va pas par ailleurs sans créer un sentiment 
d'urgence que les éditeurs du livre ont voulu partager avec le lecteur: ainsi, "l'épouvantail automobile et son corollaire, le développement périurbain insoutenable en termes spatiaux et énergétiques » (p. 16) indiquent clairement qu'en « un temps et dans un espace saturé de mobilités, pour lequel l'automobilisation individuelle a pu paraître un temps comme un absolu digne de tous les sacrifices, il est urgent de repenser l'idée de liberté traditionnellement associée à ce mode et, au-delà, de repenser l'idée même de liberté associée à toute forme de mobilité » (p. 16).

Mobilités et liberté, mobilités et démocratie, mobilités et politique : c'est autour de ces couples notionnels qui se superposent et sur ces conjonctions qui dialoguent entre elles que l'ouvrage est construit, en assumant ouvertement une haute ambition à la fois intellectuelle et civique; ambition néanmoins raisonnable, car les auteurs du livre peuvent largement bénéficier, et ils ne s'en privent pas, dans leurs efforts d'explorer la dimension politique de la mobilité des apports récents du mobility turn dans le champ des études des transports, tournant que plusieurs d'entre eux cultivent déjà ${ }^{1}$.

L'objectif général de l'entreprise annoncé dans la préface et déployé dans l'introduction générale, trois parties dotées elles-mêmes de brèves mise en perspective, se mettent à son service.

Intitulée «La mobilité en démocratie : du concept aux pratiques de gouvernement », la première partie propose d'étudier « les mobilités à travers le prisme du politique », ce qui conduit «à interroger les pratiques et formes concrètes du gouvernement des déplacements », en ouvrant du coup «un dialogue [...] avec l'analyse des politiques publiques et, plus particulièrement, avec la socio-histoire de l'action publique » (Harold Mazoyer, p. 23). Ainsi Sébastien Gardon s'intéresse à un dispositif de gestion particulière, la commission de circulation - de nature temporaire dans les années 1910 et 1920, devenue permanente et renouvelée d'année en année à partir de 1931 -, que la ville de Lyon met en place dans ses efforts pour résoudre les problèmes liés à l'irruption de la voiture dans la ville. Maxime Huré et Arnaud Passalacqua invitent à entrer dans l'univers des capitalismes urbains à l'époque de la globalisation, si chers à Dominique Lorrain : les auteurs montrent comment à partir des années 1960 et à travers une série d'innovations, de l'abribus au système de vélos en libre-service au nom de Vélo'v, la société JC Decaux a su s'imposer comme partenaire des acteurs publics de l'agglomération lyonnaise en matière d'actions urbaines, donc de mobilité. La contribution signée par Xavier Desjardins se veut une réflexion sur les échelles et espaces (institutionnels) pertinents de la régulation des mobilités. À la fin d'une analyse serrée, l'auteur conclut que « toute réforme uniquement technique et juridique serait insuffisante si l'on ne cherche pas en même temps à créer les conditions d'énonciation d'un sens partagé de l'action publique locale» (p. 95). Et à l'auteur de rencontrer le couple « mobilité et politique » par l'intermédiaire d'un nécessaire débat démocratique local. Ces trois études qui portent sur le $\mathrm{xx}^{\mathrm{e}}$ siècle et les temps présents sont précédées par une recherche historique produire par Vincent Denis. Alors que pendant longtemps l'administration du royaume français gérait de façon plus ou moins cloisonnée les déplacements de plusieurs groupes mobiles spécifiques (des mendiants, des militaires, des malades), ce n'est que durant la période 1789-1815 que, grâce à l'unification administrative et juridique du territoire de la nation, une conception générale de la mobilité, sans référence désormais à des catégories de population spécifiques, a pu voir le jour, avec le passeport, défini rigoureusement à partir de 1792, comme l'instrument clé de sa gestion. 
6 Nous transportant au-delà de l'espace strictement hexagonal, la deuxième partie de l'ouvrage («La popularisation de la mobilité et le projet démocratique ») rassemble quant à elle trois contributions, introduites par Léonard Laborie, autour de la question : «En raison de quoi la démocratie ou plutôt les projets démocratiques se sont-ils liés à la mobilité ou plutôt à des projets de mobilité ?» (p. 101). Carlos López Galviz mobilise alors les ressources de l'analyse comparative pour « comprendre dans quelle mesure la planification ferroviaire élaborée à Paris et à Londres à la fin du XIX siècle a été à la fois un des éléments et le résultat de la prise en considération de l'intérêt général dans les réflexions sur l'espace urbain des deux capitales » (p. 103). En scrutant les liens intimes tissés entre la jeune démocratie tchécoslovaque (1918-1938) et la navigation civile aérienne, Jan Oliva se pose la question plus générale : dans quelle mesure « les usages et l'appropriation par la population de la dimension symbolique des réseaux de transports constituent [...] un corolaire indispensable d'une action politique réussie» (p.118). Étienne Faugier décrit quant à lui la popularisation, terme qu'il préfère à celui de la démocratisation, de la voiture dans la partie rurale du département du Rhône, celui-ci accueillant plus d'usages et de types d'usagers que son espace urbain, entre 1890 et 1960. Alors qu'au niveau de discours, l'idéal démocratique de l'accès à l'automobilité se manifeste dès le début du $\mathrm{xx}^{\mathrm{e}}$ siècle, il faut attendre la seconde moitié du siècle pour que l'idéal se réalise pour la majorité de la population.

7 La troisième partie de l'ouvrage (« Mobilités, mobilisations et contre-mobilisations : l'ordre politique en questions ») s'organise autour du triptyque annoncé dans le titre ; il est composé de quatre textes qui informent successivement « autant sur la défense de la "cause automobile" que sur son pendant "anti-automobile" " (Stève Bernardin, p.151). S'appuyant sur un large spectre de matériaux, des rapports à l'instar du Buchanan Report (1963) du nom de son auteur, et des films comme The Italian Job (1969) de Peter Collinson, Massimo Moraglio met en évidence une série de modalités de la thématique anti-automobile dans les années 1960. Timothée Duverger s'inspire des travaux de Michel Callon et de ses collaborateurs sur la «démocratie technique » et de ceux de Pierre Rosanvallon sur la "contre-démocratie» pour explorer la «critique écologiste " de la "rationalité automobile " (se référant à Michel Foucault, l'auteur définit la rationalité comme le « pouvoir de la raison qui se manifeste comme discours de savoirs, institutions et pratiques » [p. 165]) dans les années 1970. Frédéric Héran revisite les thèses formulées il y a quarante ans par le célèbre critique de la société industrielle Ivan Illich. Si, calculs à l'appui, l'auteur conteste la thèse d'Illich, selon laquelle la « vitesse généralisée » de l'automobiliste - qui tient compte «non seulement du temps de déplacement mais aussi du temps de travail nécessaire pour payer le déplacement » (p. 178) - est inférieure à celle du cycliste, en revanche, il le rejoint dans sa critique du «monopole radical» qu'exerce l'automobile dans le champ des transports et des déplacements, et il repose à son tour la question de la légitimité démocratique d'un usage hégémonique d'un seul moyen de transport. Comme Frédéric Héran, Yves Crozet traite de la mobilité en économiste. L'auteur fournit une synthèse claire de la façon dont les économistes des transports et, dans leur sillage, les ingénieurs et les décideurs politiques ont envisagé depuis les années 1960 la question de la valeur (subjective) du temps, pour constater que la recherche de gains de temps a débouché sur un formidable allongement de la portée de nos déplacements : la relative constance des budgets temps consacrés à la mobilité conduit, en effet, à ce que la vitesse accrue ne fait pas gagner du temps mais consommer de l'espace. Des nouveaux gains de vitesse significatifs étant peu probables dans l'avenir, dans les zones denses en 
tout cas, l'auteur propose de substituer à la recherche de gains supplémentaires «le droit à ne pas perdre de temps»(p.207), droit soutenu matériellement par le formidable développement des nouvelles technologies d'information qui permettent de valoriser le temps de transport en le saturant de programmes d'activités de tous genres.

Deux courts textes prennent en charge la partie conclusive de l'ouvrage. Les trois coordinateurs dressent alors un premier bilan basé sur les textes publiés et pointent vers des recherches futures. Cotton Seiler, dans la postface, revient sur les rapports entre la mobilité et la démocratie. Tout en admettant que, «dans certaines conditions politiques et économiques et dans des contextes culturels spécifiques, la mobilité a en fait facilité la démocratie » (p. 213), l'auteur rappelle opportunément que la passion automobile est cultivée aussi par des régimes autoritaires, voire totalitaires (l'Allemagne nazie hier, la Chine (post)communiste aujourd'hui) et que, même au sein de régimes démocratiques, «l'automobilité de masse [a] charrié toute une gamme de nouveaux mécanismes d'État et d'entreprise pour le surveillance et le contrôle du citoyen ambulant » (p. 215) (pensons, en effet, aux permis de conduire, aux caméras de trafic et aux péages électroniques). Et à l'auteur de conclure que la "démocratie consiste dans le mouvement; mais le mouvement seul n'est pas constitutif de la démocratie et peut même s'y substituer et le remplacer » (p. 216).

Mené au pas de charge, cette présentation ne rend pas justice à la richesse de cet ouvrage original dans sa thématique et foisonnant dans ses analyses et résultats. Saluons donc une dernière fois les vertus de cette entreprise à la fois intellectuelle et civique, et encourageons les auteurs à la poursuivre. Regrettons quelque peu tout de même que les auteurs n'aient pas procédé à une analyse plus serrée de la notion de démocratie dont l'acception dans cet ouvrage est loin d'être stabilisée et qui varie en fonction des traditions intellectuelles mobilisées par les uns et les autres (Gauchet, Tocqueville, Rosanvallon...) : un tel travail aurait permis aux coordinateurs de faire dialoguer davantage entre elles les différentes contributions et de déboucher sur des conclusions plus tranchées.

\section{NOTES}

1. Rappelons que Mathieu Flonneau a déjà co-dirigé, avec Vincent Guigueno, un autre recueil de textes au titre significatif : De l'histoire des transports à l'histoire de la mobilité ?, Rennes, PUR, 2009. 


\section{AUTEURS}

\section{KONSTANTINOS CHATZIS}

IFSTTAR 